\title{
Isolation-By-Distance-and-Time in a Stepping-Stone model
}

\author{
Nicolas Duforet-Frebourg*, Montgomery Slatkin \\ Department of Integrative Biology, \\ University of California Berkeley, Berkeley CA 94720
}

\section{Abstract}

5 With the great advances in ancient DNA extraction, population genetics

6 data are now made of geographically separated individuals from both present

7 and ancient times. However, population genetics theory about the joint ef-

8 fect of space and time has not been thoroughly studied. Based on the clas-

9 sical stepping-stone model, we develop the theory of Isolation by Distance

10 and Time. We derive the correlation of allele frequencies between demes

11 in the case where ancient samples are present in the data, and investigate

12 the impact of edge effects with forward-in-time simulations. We also derive

13 results about coalescent times in circular/toroidal models. As one of the

14 most common way to investigate population structure is to apply principal

15 component analysis, we evaluate the impact of this theory on plots of prin-

16 cipal components. Our results demonstrate that time between samples is a

17 non-negligible factor that requires new attention in population genetics.

${ }^{*}$ corresponding author: duforetn@berkeley.edu 


\section{Introduction}

Geography plays a central role in the pattern of genetic differentiation within a species. Seminal work on describing the evolution of continuous populations was done by Wright and Malécot. They studied genetic differentiation and inbreeding in continuously distributed population (Wright, 1943 , Malécot, 1948). The resulting idea is that, under the assumption of local dispersion, genetic differentiation accumulates with distance. This pattern of genetic structure is called Isolation-By-Distance (IBD), which is detected by computing measures of differentiation such as $F_{S T}$ (Wright, 1943; Nei, 1973 Weir and Cockerham, 1984), or correlation coefficients (Malécot, 1955 Kimura and Weiss, 1964). Understanding the effect of geographic distance on population structure is an important task for population geneticist, as it is a source of neutral genetic variation (Slatkin, 1985, Rousset, 1997). Furthermore, IBD has been observed in humans and many other species (Sharbel et al., 2000; Castric and Bernatchez, 2003; Ramachandran et al. 2005: Hellberg, 2009, Karakachoff et al., 2015).

The role of geography in neutral genetic variation has been widely studied partly because of the existence of many population genetic studies of individuals living at the present time and sampled in different locations. Because of the development of methods for sequencing DNA from fossils, genomes of individuals alive at previous times are now available to bring new information about the evolutionary processes that affected a species in the past. Since the first studies of ancient DNA (aDNA) three decades ago (Higuchi et al., 1984; Pääbo, 1985), techniques to retrieve DNA molecules from ancient bones have tremendously developed (Pääbo et al., 2004).

In modern evolutionary biology, the similarity of differentiation in space and time is acknowledged (Depaulis et al., 2009; Andrello et al., 2011; Teacher et al., 2011). Theoretical developments predict the effect of time on $F_{S T}$ and related quantities (Skoglund et al., 2014). Epperson (2000) studied patterns of isolation by distance and time in ecology by using stochastic spatial time series and Identity by descent probabilities However such theoretical studies remain scarce.

The effect of separation in time can be studied using classical statistical methods in population genetics, such as principal component analysis (PCA) (Patterson et al., 2006). PCA is widely used to determine relatedness between individuals, and is a convenient way to represent geographic patterns (Novembre et al., 2008). But PCA can also capture the differentiation between ancient and modern samples: the percentage of variance explained by time can be expressed on the same scale as the percentage of variance 
explained by geography (Skoglund et al., 2014). Unfortunately, PCA does

not give a complete picture of how differentiation quantities such as $F_{s t}$ and correlations of allele frequencies evolve in time and space.

In this article we generalize the theory of IBD to allow for difference in the times at which different individuals are sampled. We call this the theory of isolation by distance and time (IBDT). We base our work on the stepping-stone model of Kimura (1953) and add to the theoretical results known for this model (Kimura and Weiss, 1964, Weiss and Kimura, 1965 , Maruyama, 1971a; Nagylaki, 1983; Cox et al. 2002; De and Durrett, 2007). We start by briefly reviewing the original results for the infinite steppingstone model at equilibrium and the decay of correlation of allele frequencies with distance. Then, we extend the original work to derive the correlation between individuals separated by distance and time. We perform simulations that show the validity of the analytic results, even in the case of a finite number of populations where some demes are subject to edge effect. We also derive the expected coalescence times between samples separated by time and space in circular and toroidal models (Slatkin, 1991, 1993). Finally we consider the consequences of IBDT on PCA in the common case of a dataset made of a large proportion genomes from present-day individuals and few ancient genomes.

\section{The stepping-stone model}

The stepping-stone model describes the distribution of allele frequencies in an infinite set of demes in different locations of the space represented by Cartesian coordinates. We start by describing the 1-Dimensional case. Let $p(k)$ be the frequency of one allele at a bi-allelic locus in population $k$ and $\bar{p}$ be the overall allele frequency. In each generation, $p(k)$ is updated with the following three steps (Crow et al., 1970):

- Exchange a proportion $m_{i}$ of migrants with demes at a distance $i$.

- Exchange a proportion $m_{\infty}$ of migrants with a deme that has fixed allele frequency $\bar{p}$. The meaning of this step is discussed later.

- Sample gametes of the next generation in the population.

In the case considered by Kimura and Weiss (1964), migrants are exchanged only between neighboring locations in the first step, so that $m_{i}=$ $0, i>1$. The second step consists of exchanges of migrants with an external population at rate $m_{\infty}$. This event is equivalent to reversible mutations 
102

occurring. The formulation of the model states that every locus is bi-allelic, and the number of loci is fixed. As a consequence, the mutation model is a reversible mutation model with probability $m_{\infty}$, and $m_{i}>>m_{\infty}$. Random sampling of step 3 is represented by a random change in the allele frequency $\epsilon(k)$, with $E[\epsilon(k)]=0$, and $E\left[\epsilon(k)^{2}\right]=p(k)(1-p(k)) / 2 N_{e}$, where $N_{e}$ is the effective population size of a deme (Wright, 1940; Kimura and Crow, 1963).

Our interest is in the changes in allele frequency in one generation. We consider $\tilde{p}(k)=\bar{p}-p(k)$, the deviation from the average frequency. Given these three steps,

$\tilde{p}^{\prime}(k)=\left(1-\sum_{i=1}^{\infty} m_{i}-m_{\infty}\right) \tilde{p}(k)+\frac{m_{1}}{2}(\tilde{p}(k-1)+\tilde{p}(k+1))+\frac{m_{2}}{2}(\tilde{p}(k-2)+\tilde{p}(k+2))+\cdots+\epsilon(k)$.

To simplify the notation, we define the operators $S$ and $L$,

$$
S \tilde{p}(k)=\tilde{p}(k+1), S^{-1} \tilde{p}(k)=\tilde{p}(k-1),
$$

$$
L=\left(1-\sum_{i=1}^{\infty} m_{i}-m_{\infty}\right) S^{0}+\sum_{i=1}^{\infty} \frac{m_{i}}{2}\left(S^{i}+S^{-i}\right)
$$

so that,

$$
\tilde{p}^{\prime}(k)=L \tilde{p}(k)+\epsilon(k) .
$$

The quantity of interest in this model is the correlation of allele frequencies between two demes at locations $k_{1}$ and $k_{2}$. Let $r(k)$ be the correlation coefficient of allele frequencies between populations that are $k$ steps apart. Assuming equilibrium, we have

$$
r(k)=\frac{\rho(k)}{\rho(0)}=\frac{E\left[\tilde{p}\left(k_{1}\right) \tilde{p}\left(k_{2}\right)\right]}{\rho(0)}=\frac{E\left[L \tilde{p}\left(k_{1}\right) L \tilde{p}\left(k_{2}\right)\right]}{\rho(0)},
$$

where $\rho(k)$ is the covariance in frequencies in demes $k$ steps apart. The mathematical treatment of equation (5) by Weiss and Kimura (1965) using the spectral representation of a correlation (Doob, 1953) gives the general formula

$$
r(k)=\frac{C}{2 \pi} \int_{0}^{2 \pi} \frac{\cos (k \theta) d \theta}{1-\left[\sum_{i=0}^{\infty} m_{i} \cos (i \theta)\right]^{2}},
$$

where $C$ is the normalizing constant.

Equation (6) can be approximated by an exponential function of $k$ :

$$
r(k)=e^{-\sqrt{\frac{2 m_{\infty}}{m_{1}}} k} .
$$


This simple formula conveys the important idea that in one dimension, the correlation of allele frequencies between populations decays exponentially with distance. In the 2-Dimensional and 3-Dimensional cases, the correlation function is more difficult to approximate. Using modified Bessel function, it is shown that correlation at a given distance is lower in these cases than in the 1-Dimensional case (Weiss and Kimura, 1965).

\section{Isolation-by-Distance-and-Time}

\section{$3.1 \quad 1$-Dimensional case}

We are here interested in the case where genetic samples are collected from demes that are in different locations and at different times (measured in generations). Let $\rho(k, t)$ be the covariance between allele frequencies of two demes separated by $k$ steps and $t$ generations. We denote the coordinates of these demes by $\left(k_{1}, t_{1}\right)$ and $\left(k_{2}, t_{2}\right)$, and the deviations in allele frequencies $\tilde{p}\left(k_{1}\right)^{\left(t_{1}\right)}$ and $\tilde{p}\left(k_{2}\right)^{\left(t_{2}\right)}$. Since we assume the distribution of allele frequencies is stationary in both time (equilibrium distribution) and space (all migration rates are equal), we can consider these coordinates to be $(0,0)$ and $(k, t)$ with no loss of generality. Following previous notation

$$
\rho(k, t)=E\left[\tilde{p}\left(k_{1}\right)^{\left(t_{1}\right)} \tilde{p}\left(k_{2}\right)^{\left(t_{2}\right)}\right]=E\left[\tilde{p}(k)^{(t)} \tilde{p}(0)^{(0)}\right] .
$$

To characterize the evolution of the covariance between allele frequencies with respect to time $t$, we iteratively apply the operator $L$ defined in equation (3). This operation describes the potential trajectories of an allele, and results in a quantity similar to a propagator. This process leads to

$$
\rho(k, t)=L^{t} \rho(k)
$$

with $\rho(k)=\rho(k, 0)$ (see Appendix A).

Let $r(k, t)$ be the correlation between allele frequencies of two demes separated by $k$ steps and $t$ generations, equations (5) and (9), combined with the general formula of equation (6) gives

$$
r(k, t)=\frac{C}{2 \pi} \int_{0}^{2 \pi} \frac{\left[\sum_{i=0}^{\infty} m_{i} \cos (i \theta)\right]^{t} \cos (k \theta) d \theta}{1-\left[\sum_{i=0}^{\infty} m_{i} \cos (i \theta)\right]^{2}} .
$$

and the constant $\mathrm{C}$ is set such that $r(0,0)=1$ (Appendix B).

This equation reduces to

$$
r(k, t)=\frac{C}{2 \pi} \int_{0}^{2 \pi} \frac{\left[1-m_{1}-m_{\infty}+m_{1} \cos (\theta)\right]^{t} \cos (k \theta) d \theta}{1-\left(1-m_{1}-m_{\infty}+m_{1} \cos (\theta)\right)^{2}}
$$


in the standard stepping-stone model, where demes only exchange migrants with their closest neighbors at rate $m_{1} / 2$. An exact formula for this integral can be calculated and is notable for its size and lack of utility (Appendix C).

One noteworthy feature of equation (10) is that the decay of the correlation with time is not affected by the effective population size $N_{e}$. This result is different from what is expected for an isolated population: the level of differentiation as a function of the number of generations separating two samples is larger when the effective population size is small, reflecting the increased magnitude of genetic drift. However, in the particular case of an equilibrium stepping-stone model, the covariance of allele frequencies between the demes is not a function of the effective population size, a result already known in the spatial context (see equation (7)) (Kimura and Weiss, 1964). This result becomes clear when considered in terms of coalescence times. Between the time the first and second samples are taken, the trajectory of the first sample depends only on the migration process. There is no possibility of coalescence.

\subsection{Two dimensions and more.}

So far, we have focused on the 1-Dimensional case for the sake of simplicity. However, it is important to investigate the decay in higher dimensions as it is common in practice to have samples taken from a 2-Dimensional or even 3-Dimensional habitat. The general formula for the correlation in higher dimensions can be obtained with no more theoretical development. In their work on the stepping-stone model, Kimura and Weiss derived a general formula for the correlation that can be extended to any number of dimensions. In their work they only gave approximations for 1,2 or 3 dimensions as these are the practical cases. Using general formula (3.11) of Weiss and Kimura (1965), we can write the correlation 10 in 2 dimensions

$r\left(k_{1}, k_{2}, t\right)=\frac{C_{2}}{(2 \pi)^{2}} \int_{0}^{2 \pi} \int_{0}^{2 \pi} \frac{\left[\sum_{i_{1}=0}^{\infty} \sum_{i_{2}=0}^{\infty} m_{i_{1} i_{2}} \cos \left(i_{1} \theta_{1}\right) \cos \left(i_{2} \theta_{2}\right)\right]^{t} \cos \left(k_{1} \theta_{1}\right) \cos \left(k_{2} \theta_{2}\right) d \theta_{1} d \theta_{2}}{1-\left(\sum_{i_{1}=0}^{\infty} \sum_{i_{2}=0}^{\infty} m_{i_{1} i_{2}} \cos \left(i_{1} \theta_{1}\right) \cos \left(i_{2} \theta_{2}\right)\right)^{2}}$.

The generalization to obtain the correlation in $n$ dimensions is straightforward (Appendix D).

We perform a numerical integration of equation 12 to investigate the decay of correlation with distance and time in one dimension and higher. Correlation decreases as a function of distance and time in 1, 2 and 3 dimensional models (Figure 1). In addition, for equal values of the migration 
and mutation rates the correlation decrease is much larger with respect to time and geography in higher dimension models, consistently with previous results (Maruyama, 1970a, 1971a). Numerical integration is done using the $R$ package cubature.

\subsection{Simulations in one dimension and two dimensions.}

When considering realistic examples, a finite number of demes is present in the data. As a consequence, correlation patterns are affected by the proximity of the edge of the sampling scheme (Maruyama, 1970b). Another effect of the finite number of demes is that the overall allele frequency can drift away from the theoretical allele frequency. An alternative is to consider a finite, non-circular model, and to deal with edge issues independently (Felsenstein, 2015). To investigate to which extent the analytic theory developed in the previous section is valid in a finite stepping-stone model with temporal sampling, we perform simulations.

Backward in time simulation software such as $m s$ (Hudson, 2002), or fastsimcoal (Excoffier and Foll, 2011), are usually used to investigate IBD in a stepping-stone models (Novembre et al., 2008). Temporal sampling can be investigated in such model by simulating gene trees where lineages from isolated demes are joined to the stepping-stone demes at a chosen time in the past (Skoglund et al., 2014). Mutations are then randomly placed on the gene tree. Such a simulation is needed to understand the influence of time and distance on genetic differentiation, but does not precisely reproduce the process of the above model which assumes reversible mutation rather than the infinite site model. The infinite site model does not have a true equilibrium for any one site, only a pseudo-equilibrium.

We wrote a $\mathrm{C}$ program that performs forward in time simulations. The simulation program precisely follows the model presented in the previous section. At the initial time, the allele frequencies in all the demes are equal to the allele frequencies in the outside infinite-sized population. Then the program runs for a large number of generations until the stationary distribution of the allele frequencies is reached.

In the 1-Dimensional case, we simulate 100 demes. For the 2-Dimensional case, we simulate a total of 2500 demes on a $50 \times 50$ grid. We assume all the demes have the same effective population size. We sample the allele frequencies at several times in the past. Correlation between demes fit very closely the theory of equations (11) and (12) provided that demes are taken sufficiently far away from the edge of the grid (Figure 2). The edge effect directly increases the correlation between demes, and is present when com- 
paring present and ancient samples. In both 1 and 2 dimensions, the edge effect disappears in the simulations (Figure 3). As predicted by Maruyama, the edge effect is less strong with lower migration rates.

\section{Coalescence times}

\subsection{Coalescence times in one dimension}

Coalescence times in a stepping-stone model can be derived under some assumptions. In particular, we consider a case with migration only between neighboring demes and low mutation rate. Expected coalescence times between genes that are in different demes is a function of the locations of these demes. These coalescence times are of interest because they closely related to $F_{S T}$ and coefficients of identity-by-descent (Slatkin, 1991). Under the assumption of a circular 1-Dimensional stepping-stone model with $n_{d}$ demes, two genes $A_{1}$ and $A_{2}$ have an expected coalescence time

$$
E\left[T_{A_{1} A_{2}}\right]=2 N_{e} n_{d}+\left(n_{d}-k\right) \frac{k}{2 m},
$$

where $N_{e}$ is the effective population size per deme, $m$ the migration rate between neighboring demes (previously $m_{1}$ ), and $k$ is the distance between the two demes (Slatkin, 1991). Considering a circular arrangement of the demes makes the analysis simpler, as only the distance between the demes matters, and there are no edge effects. In addition it has been shown that linear/planar and circular/toroidal stepping stone models are very similar when considering population away from the edges (Maruyama, 1971a b). To study a case similar to the infinite stepping-stone model, we assume $n_{d}$ is large.

We extend the previous theoretical result in the case where two genes are sampled at different times. Let us assume that the sampled genes are in population $k_{1}$ and $k_{2}$. The number of generations between the two sampling times is $t=t_{1}-t_{2}$, and we assume, with no loss of generality, that $t_{1}=0$ and $t_{2}=t$ generations in the past. The coalescence process between these two genes can be divided in three phases. The first phase corresponds to the genealogy that traces back to the ancestor of the present gene, called $A_{1}^{(t)}$, at generation $t$. This ancestor is in population $k_{1}^{(t)}$. The two other parts correspond to the time until the coalescence event between $A_{1}^{(t)}$ and $A_{2}$. They are respectively the time until the gene $A_{1}^{(t)}$ and $A_{2}$ are in the same deme, then the time to the common ancestor of these two genes. This 
part has already been described, and the expectation is given in equation (13) (Slatkin, 1991). The expected coalescence time between $A_{1}$ and $A_{2}$ is then written

$$
E\left[T_{A_{1} A_{2}}\right]=t+E\left[T_{A_{1}^{(t)} A_{2}}\right] .
$$

The variable $T_{A_{1}^{(t)} A_{2}}$ is the coalescence time between a random gene in the unknown population $k_{1}^{(t)}$ and a random gene in population $k_{2}$. To represent the uncertainty about the population $k_{1}^{(t)}$, we derive the probability distribution of the position $k_{1}^{(t)}$ at time $t$, given position $k_{1}$ at time 0 . Using this probability distribution we rewrite the expectation (14) as

$$
E\left[T_{A_{1} A_{2}}\right]=t+\sum_{x=0}^{n_{d}-1} E\left[T_{A_{1}^{(t)} A_{2}} \mid k_{1}^{(t)}=x\right] p\left(k_{1}^{(t)}=x\right) .
$$

To describe the probability distribution of position $k_{1}^{(t)}$ at time $t$ given that a gene is in population $k_{1}$ at time 0 , we consider a random walk with transition matrix

$$
M=\left(\begin{array}{cccccc}
1-m & \frac{m}{2} & 0 & \ldots & 0 & \frac{m}{2} \\
\frac{m}{2} & 1-m & \frac{m}{2} & \ldots & 0 & 0 \\
0 & \frac{m}{2} & 1-m & \ldots & 0 & 0 \\
\ldots & & & & & \\
\frac{m}{2} & 0 & 0 & \ldots & \frac{m}{2} & 1-m
\end{array}\right) .
$$

Using standard results about Markov chains (Ross et al. 1996), we know that the vector of probabilities for the position at time $t, P_{k_{1}^{(t)}}$ is expressed such as

$$
P_{k_{1}^{(t)}}=M^{t} P_{k_{1}}
$$

with $P_{k_{1}}$ is the initial probability distribution of gene $A_{1}$ 's position. The initial probability distribution is trivial and $P_{k_{1}}$ is a vector of 0 with a 1 in the $k_{1}^{t h}$ entry. Exact formula for this matrix power can be obtained using tridiagonal matrix properties (Al-Hassan, 2012). However we can also express an approximation for the probability distribution of this process at time $t$. This random process is symmetrical, centered in $k_{1}$, and using classical results about Brownian motion, has a variance proportional to $t$. We can approximate the probability distribution by a Normal distribution, and

$$
P\left(k_{1}^{(t)}=x \mid k_{1}\right)=\mathcal{N}\left(x ; k_{1}, m t\right) .
$$


The accuracy of this approximation can be verified with simulations using equation (17). The approximation is relevant for sufficiently large values of $t$, depending on the migration rate. The expected coalescence time in a 1-Dimensional circle can then be written

$$
E\left[T_{A_{1} A_{2}}\right]=2 N_{e} n_{d}+t+\frac{1}{\sqrt{2 \pi m t}} \int_{0}^{n_{d}-1}\left(n_{d}-\left|x-k_{2}\right|\right) \frac{\left|x-k_{2}\right|}{2 m} e^{-\frac{\left(x-k_{1}\right)^{2}}{2 m t}} d x .
$$

Coalescence time between genes is an increasing function of distance and time between demes (Figure 44. Asymptotically, when $t$ is large, the expected time for two genes to be in the same population can be approximated by a linear function of time between the samples (Figure 4). The right part of equation (19) is the integral of a product of a positive function that depends only on the distance between demes and a Gaussian kernel with variance $m t$. As the time gets large, relatively to $m$, the Gaussian kernel becomes flat, and the integral is almost constant (Figure 4). In practice, this implies that in a population at equilibrium, the geography does not matter when the sample is very old.

\subsection{Coalescence times in two dimensions}

In the case of a 2-Dimensional habitat with $n_{d 1} \times n_{d 2}$ demes, the expected coalescence time between two genes $A_{1}$ and $A_{2}$ is

$$
E\left[T_{A_{1} A_{2}}\right]=N_{e} n_{d 1} n_{d 2}+\frac{S(i, j)}{2 N_{e} m},
$$

where $S(i, j)$ is a function of $i$ and $j$, the number of demes between the two genes. We assume in this case that the migration in each direction is the same.

Using the same conditioning as in equation (14), we can derive the expectation for the coalescence time of genes $A_{1}$ in population $\boldsymbol{k}_{1}$ and $A_{2}$ in population $\boldsymbol{k}_{2}, t$ generations in the past. We have

$$
E\left[T_{A_{1} A_{2}}\right]=t+\sum_{x_{1}=0}^{n_{d 1}-1} \sum_{x_{2}=0}^{n_{d 2}-1} E\left[T_{A_{1}^{(t)} A_{2}} \mid \boldsymbol{k}_{1}^{(\boldsymbol{t})}=\left(x_{1}, x_{2}\right)\right] p\left(\boldsymbol{k}_{\mathbf{1}}^{(\boldsymbol{t})}=\left(x_{1}, x_{2}\right)\right)
$$

The probability distribution of the position of gene $A_{1}$ at time $t, \boldsymbol{k}_{1}^{(\boldsymbol{t})}$ is known using the same random walk as in the 1-Dimensional case. The distribution can be approximated by a bivariate Normal distribution with 
mean $\boldsymbol{k}_{1}$, and covariance matrix $\Sigma$, where $\Sigma$ is diagonal with terms $m t / 2$ in the diagonal. In the anisotropic case where migration rate would be different in the two dimensions, $m_{1}$ and $m_{2}, \Sigma$ would have $m_{1} t$ and $m_{2} t$ as diagonal terms. The evaluation of this function for samples separated in distance and time shows a similar pattern to the 1-Dimensional case (Figure 4). However for a same migration rate, the expected times for two genes to be in the same deme in the 2-Dimensional toroidal model are smaller than in the 1Dimensional circular model. Then, if there is the same number of demes, with same effective population sizes, e.g. $n_{d} N_{e}=n_{d 1} n_{d 2} N_{e}$, the expected coalescence times are smaller in the 2-Dimensional case. This result is already known when comparing samples taken at the same generation and remains true when $t$ is positive (Slatkin, 1993).

\section{Connection with PCA}

Because there is a close connection between PCA and coalescence times (McVean, 2009), our results are relevant to using PCA to compare ancient and modern samples. PCA is a useful way to represent the main axes of variation in the data and has proven to be a powerful tool to infer genetic relationships when applied to ancient DNA data(Skoglund et al., 2012; Haak et al. 2015).

\subsection{Ancient samples are shrunk towards 0 .}

In population genetics, PCA is usually performed by computing the eigenvectors, and eigenvalues of the matrix of covariances in the genotypes of different individuals. Although there are other ways to compute principal components, this one is convenient in population genetics because the number of variables is usually larger by several orders of magnitude than the number of samples. The effect of differences in the sampling times can be evaluated using the dependence of the covariance matrix described by equation (10). To illustrate, consider a 2-Dimensional even repartition of $10 \times 10$ demes, and ancient samples taken in several randomly chosen demes at $t=1000$ generations in the past (Figure 5A). By calculating the theoretical covariance matrix and its first two eigenvectors, we obtain the first two principal components that reproduce geography of the demes (Novembre et al., 2008; Engelhardt and Stephens, 2010). Figure (5B) shows that principal components mimic the geography of the present demes, but ancient demes are not superposed on the corresponding present-day sample 
from the same deme. Instead, ancient samples move towards the center of the first and second principal components.

Using 100 demes from a 1-Dimensional simulation described above, we apply PCA to the allele frequencies at the 6000 simulated loci. To remove the edge effect, we simulate 200 demes, and consider only the 100 demes in the center. We also include allele frequencies from past generations for several demes. PC1 shows the 1-Dimensional pattern of isolation-by-distance as expected, and ancient samples are closer to 0 (Figure 6A). The distance between the scores of ancient individuals and the center of the principal component decreases as the sampling time increases. In practice, the true allele frequencies are not known, and the covariance matrix is estimated on individuals. When working with sampled individuals instead of allele frequency, the same pattern is still visible. A subsampling of 10 diploid individuals for each deme at the present time, and 1 diploid individual for each ancient deme shows the same shrinkage of PC scores for ancient individuals (Figure $6 \mathrm{~B})$.

When applying PCA on allele frequencies from the 2-Dimensional simulations, the time effect is visible on the first two components. We study the case of a $10 \times 10$ grid, with no edge effects, and ancient samples taken from 4 demes at different times in the past (Figure $6 \mathrm{C}$ ). The first and second principal components reproduce the geography of the samples, and the ancient samples are moved towards the center of the plot (Figure 6D).

This shrinkage effect of time can be understood considering the shape of the covariance function. The first and second principal components represent the 2-Dimensional Isolation-By-Distance pattern. This pattern causes the covariance matrix at time $t=0$ to have a "block Toeplitz with Toeplitz blocks" form (Novembre and Stephens, 2008). However the pairwise covariance between present-day individuals $(t=0)$ and between ancient and present-day individuals $(t>0)$ does not have the same shape (Figure 1). Equation (10) implies that in a stepping-stone model the covariance as a function of distance flattens when comparing present and ancient individuals. As a consequence, the scores of ancient samples are moved towards the center of the principal components reproducing the local correlation pattern. Thus ancient samples can cluster with present samples at different locations, even in an equilibrium stepping-stone model.

\subsection{One component for the time differentiation}

Links between PCA and population genetics quantities, such as coalescence times and $F_{S T}$ have been studied (McVean, 2009, Duforet-Frebourg et al. 
2015, Baran and Halperin, 2015) and show that these values can be estimated from principal components. In the 2-population case, McVean (2009) showed that the distance between individuals on the appropriate principal component is approximately a linear function of the square root of the time, $\Delta$, until the lineages of the two individuals are in the same deme. If there are ancient and present samples, they can be considered as two groups, and $\Delta$ is the time corresponding to the first two parts of the coalescence process between the lineages, described in the previous section. The time separating the individuals is a source of variance important enough to be reflected in the principal components (Skoglund et al., 2014). In this case, one component separates the two groups and the distance between groups is approximately proportional to $\sqrt{\Delta}$. In Appendix $\mathrm{E}$, we compute the expectation of $\Delta$ if there are several present-day and one ancient individuals sampled.

We analyze the case with 50 contiguous populations sampled from a circular 1-Dimensional stepping-stone model with $n_{d}=1000$. We assume $m_{1}=0.1$, and one deme is sampled in the past. We apply PCA by computing the eigenvectors of the individuals correlation matrix. The first principal component represents the IBD pattern between the present demes (Figure $7 \mathrm{~A}$ ). The second principal component corresponds to the differentiation between the ancient deme, and the present demes. The average distance on PC2 between the two groups (present and ancient) is an increasing function that can be approximated by a linear function of the square root of $\Delta$ (Figure 7B).

\section{Conclusions and discussion}

We have generalized the Kimura-Weiss theory of a stepping-stone model to the case where samples are taken at different times, a theory we call Isolation-by distance-and-time (IBDT). The correlation between individuals decreases as a function of both geographic distance and time. This result is accentuated in higher dimensions. When considering IBDT patterns, the edge effect applies when considering a linear model with a finite number of demes, similarly to the standard stepping-stone model. However simulations shows that in both 1 and 2 dimensions, this effect vanishes at a rate depending of the migration rate. We have also derived the expected coalescence times under the assumption of a circular, or toroidal model and low mutation rate. As the time between samples increases, the coalescence time between samples can be approximated by a linear function of time.

The connection between IBDT theory and PCA is of interest as it gives 
insights about what to expect from the PC plots that compare ancient and present-day samples. When considering only principal components reproducing geography, scores of the ancient samples may not cluster with the population at the same location. Such a result can occur even in the case of a population at equilibrium in a stepping-stone model, with no complex demographic history. This behavior of PCA is important to note as it could result in the inference of a non-existent ancient demographic event. The genetic differentiation created by time can be observed on another principal component. An important question that remains is in which conditions the proportion of variance explained by time is larger than the proportion of variance explained by Geography. In this unlikely event, the first principal component would not reproduce geography of the samples but rather the time line of the samples.

The limitations of PCA to investigate population structure in a spatiotemporal context highlights the need for new theoretical developments to analyze population structure when present-day and ancient samples are combined. This is especially apparent when considering the complex demographic scenarios already inferred about the history of modern humans (Pickrell and Reich, 2014). Important theoretical work has already been done to test specific hypothesis (Durand et al., 2011; Loh et al., 2013). Another way to test different past demographic events is with intensive simulation procedures, such as Approximate Bayesian Computations (Beaumont et al. 2002; Csilléry et al., 2010). In this case, theoretical developments on mechanistic models such as the stepping-stone model are important to perform simulations efficiently (Baird and Santos, 2010).

We studied the classical stepping-stone model under the assumptions of a stationary distribution of the allele frequencies in both time and space. These assumptions are not valid in all cases. The time-stationary distribution is not reached when recent events such as range expansions occurred, causing asymmetry in the site frequency spectrum (Hallatschek et al., 2007, Peter and Slatkin, 2013). Spatial non-stationarity and anisotropy can occur when migration pattern is uneven between all populations, or migration is favored in one direction (Jay et al., 2013: Duforet-Frebourg and Blum, 2014 , Petkova et al. 2014). The correlation of allele frequencies is then not only a function of space and time, but also of the locations of each deme in the habitat.

A stepping-stone model is not the only model to describe spatial population structure. As an alternative to discrete models, continuous models can also be considered to study evolutionary processes (Maruyama, 1972, Barton et al., 2002, 2010). Isolation-by-Distance-and-Time can be studied in a 
445 continuous framework. In the same way, results about coalescence times in

446 a stepping-stone model can be connected to previous theory on coalescence

447 in a continuous population (Wilkins and Wakeley, 2002).

\section{${ }_{448}$ Acknowledgement}

449 This work was supported by NIH grant R01-6M40282 to M. Slatkin. 

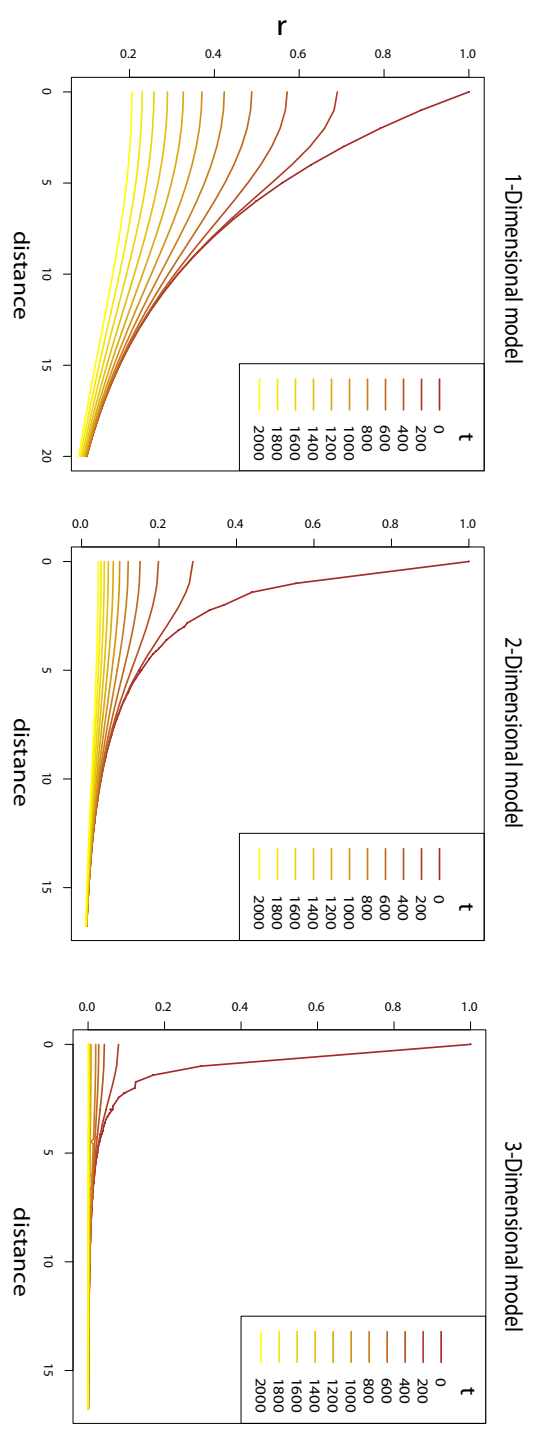

Figure 1: Correlation as a function of distance between demes $k$ steps appart in 1,2 and 3-Dimensional models. The correlation is evaluated for different number of generations $t$ between the demes. The migration and mutation rates are used for all models, and $m_{1}=.01$ and $m_{\infty}=4.10^{-4}$. 

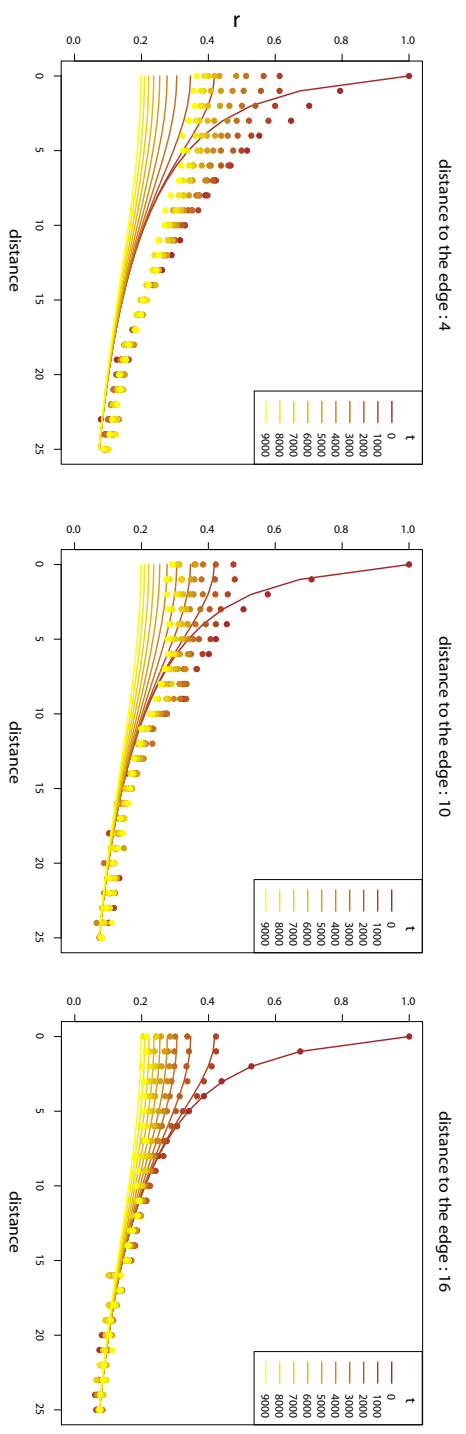

Figure 2: Comparison between theoretical results and simulations in the 2 dimensional case with $m_{1}=.02$ and $m_{\infty}=10^{-5}$. The solid lines represent the theory prediction. The dots represent the simulation results evaluated for demes at a distance 4,10 or 16 from the edges. Since in the simulations several pairwise comparisons between demes have the same distance in space and time, we keep the median of these pairwise correlations. 

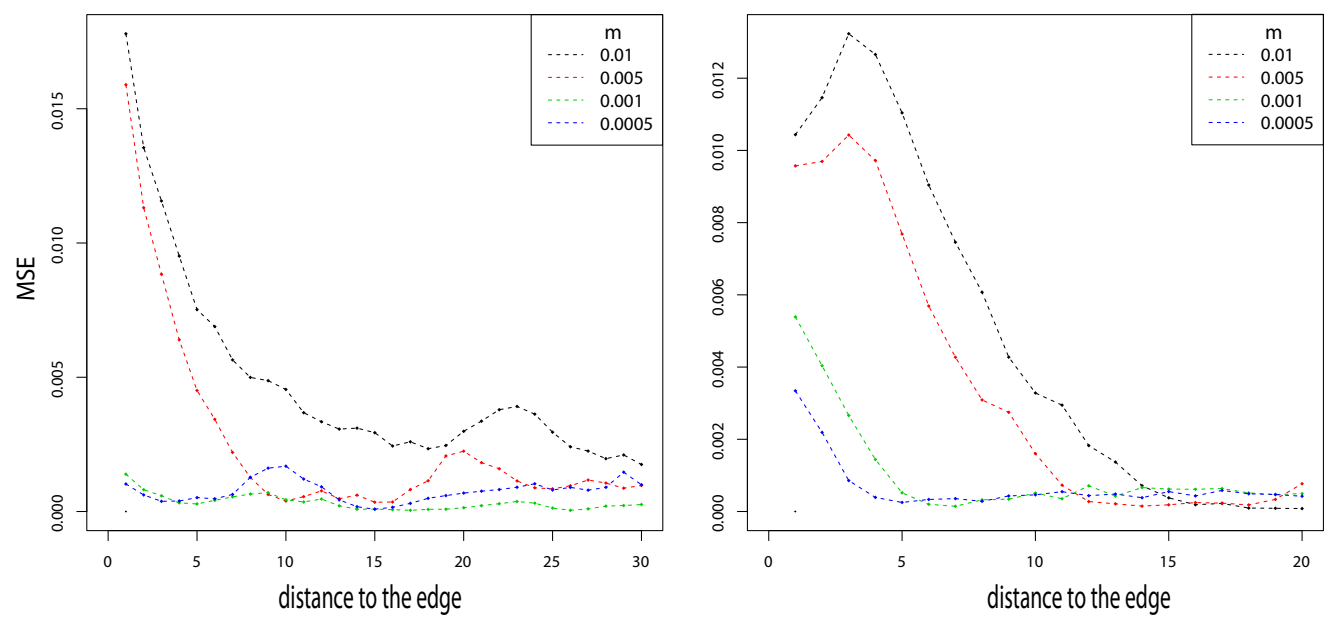

Figure 3: Mean squarred error between simulations and theory in 1 and 2 Dimensions as a function of the distance to the edge. The error is evaluated for $m_{\infty}=10^{-5}$ and $m_{1}=.01, .005, .001, .0005$. 

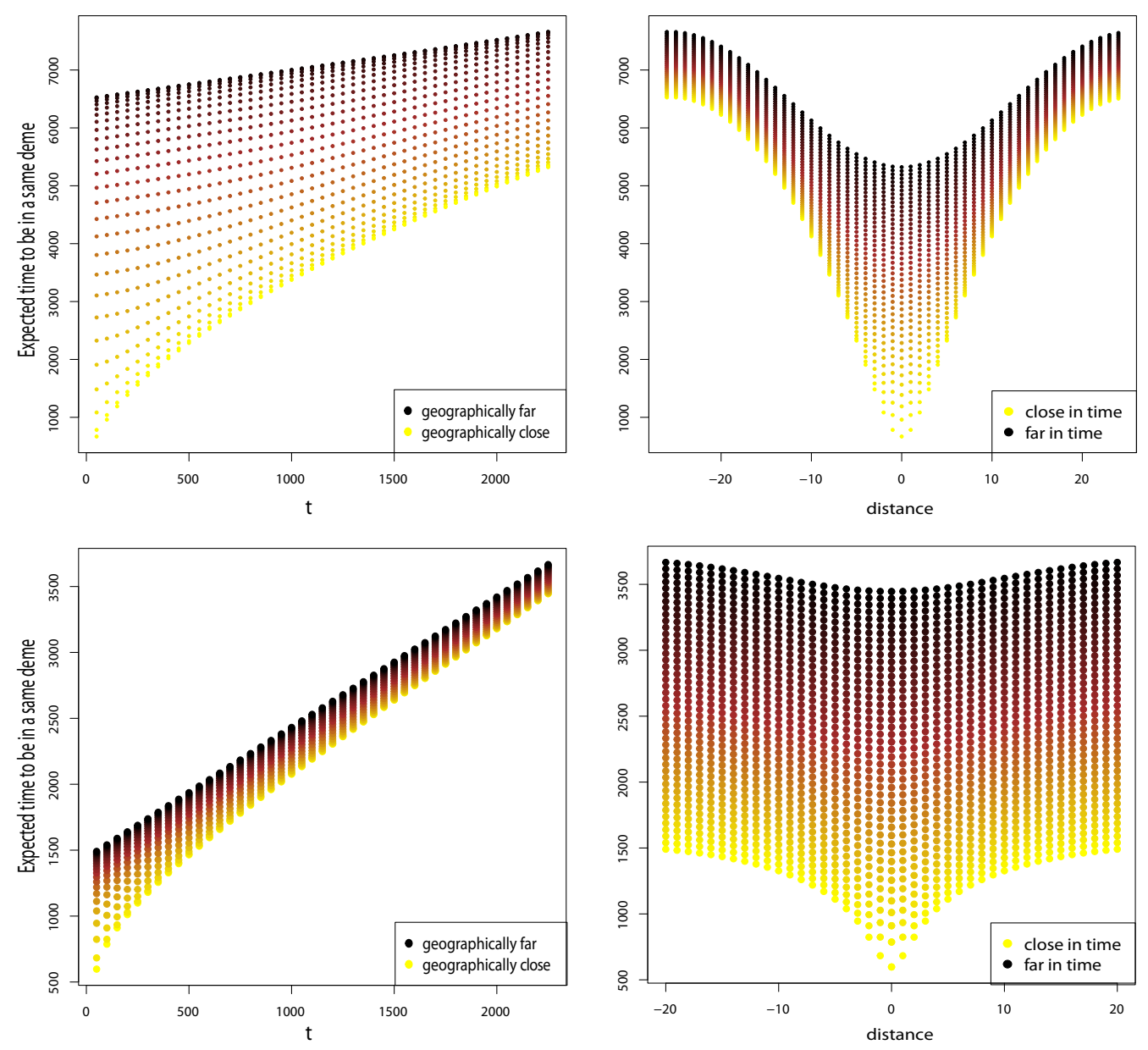

Figure 4: Top row: Expected time for two genes to be in a same deme in a 1-Dimensional circular stepping-stone model with $N_{e}=100, m=.01$, and $n_{d}=51$ demes. Bottom row: Expected time for two genes to be in a same deme in a 2-Dimensional toroidal stepping-stone model with $N_{e}=100$, $m=.01$, and $n_{d}=51 \times 51$ demes. Left column: Expected times as a function of the time between the samples. Colors indicate the geographic distance between samples. Right column. Expected times as a function of geographic distance between the samples. Colors indicate the time between samples. Sampling consists in 45 time points evenly separated by 50 generations. 

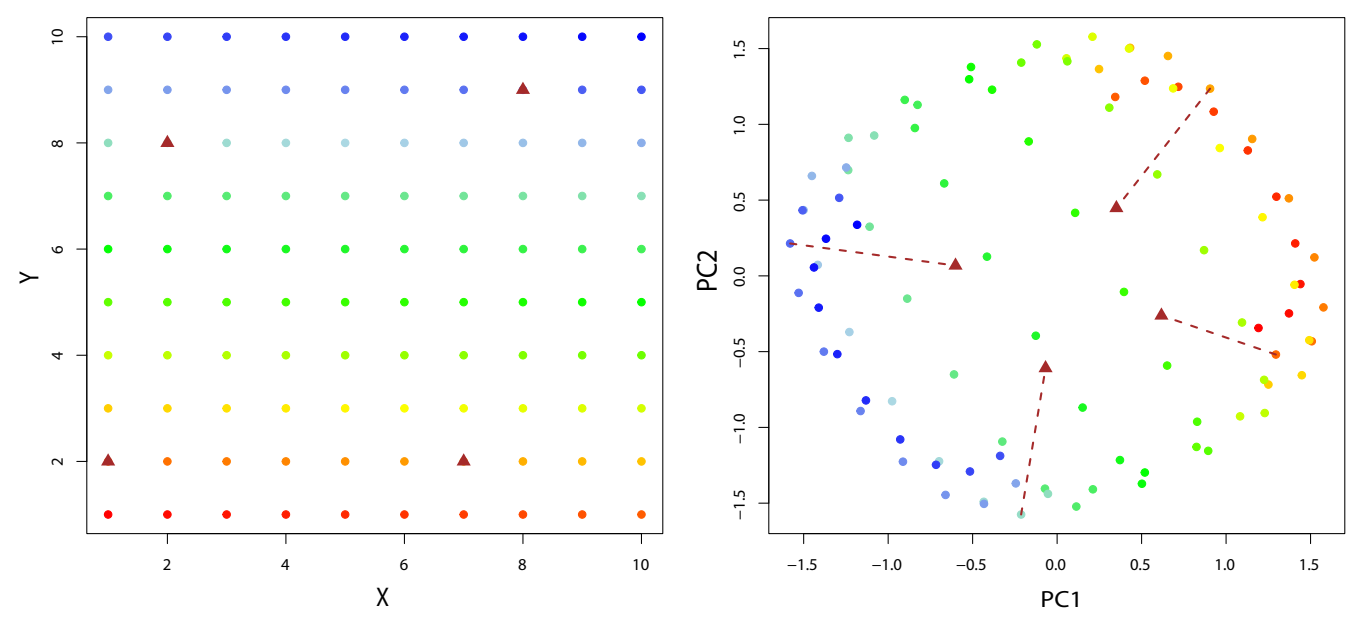

Figure 5: Panel A. Sampling scheme of a $10 \times 10$ grid of demes. Brown triangles represent demes where ancient individuals are sampled 1000 generations in the past. Panel B. First 2 eigenvectors of the covariance matrix between populations of Panel A. Parameters used are $m_{1}=.01$ and $m_{\infty}=10^{-5}$. Color code is the same as in Panel A. Brown arrows start from the position of the present deme where an ancient sample is taken, and end where the ancient sample is projected on the principal components. 
A
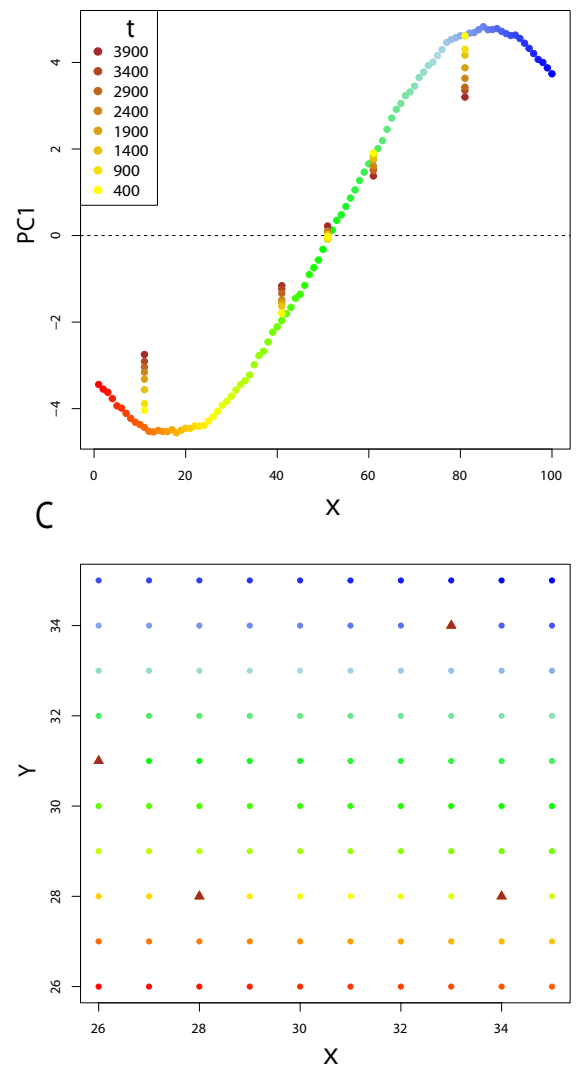

B
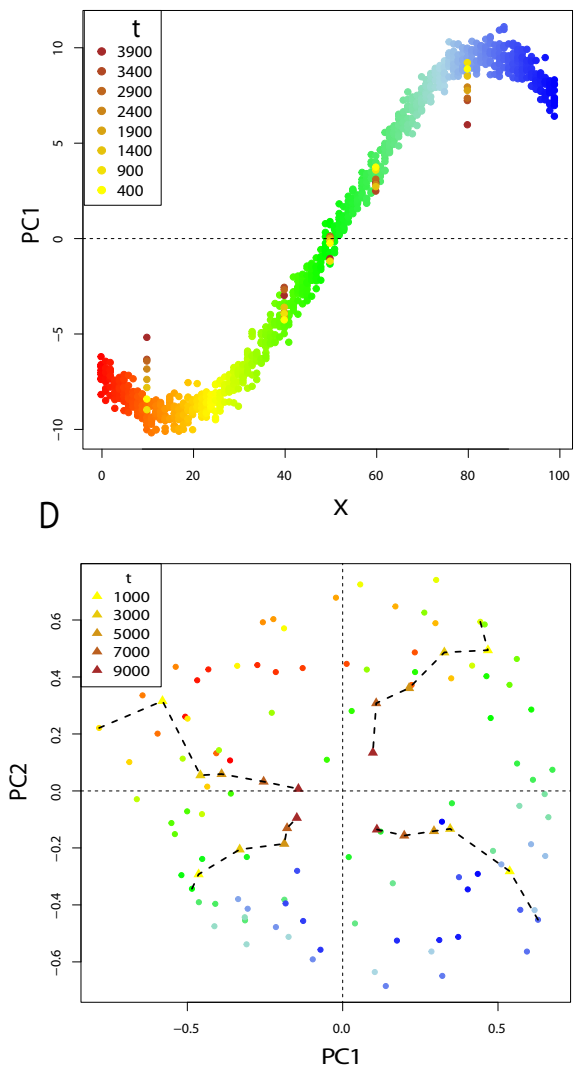

Figure 6: Panel A. First principal component for the 1-Dimensional simulation described above, with $m_{1}=.01$ and $m_{\infty}=4 \cdot 10^{-5}$. PCA is performed on allele frequency data from each of the 100 demes, and ancient allele frequencies are taken in 5 populations at 8 times in the past. Panel B. First principal component for the 1-Dimensional simulation described above. In each deme, 10 diploid individuals are sampled at the present time. One diploid individual is sampled in 5 demes at 8 times in the past. Panel C. Sampling scheme of a $10 \times 10$ grid of populations. Demes marked by a triangle are demes where ancient individuals were sampled. Panel D. plot of $P C 1$ and $P C 2$ for the 2-Dimensional simulation with $m_{1}=.001$ and $m_{\infty}=10^{-5}$. Ancient samples are taken at different times in the past for 4 demes. 

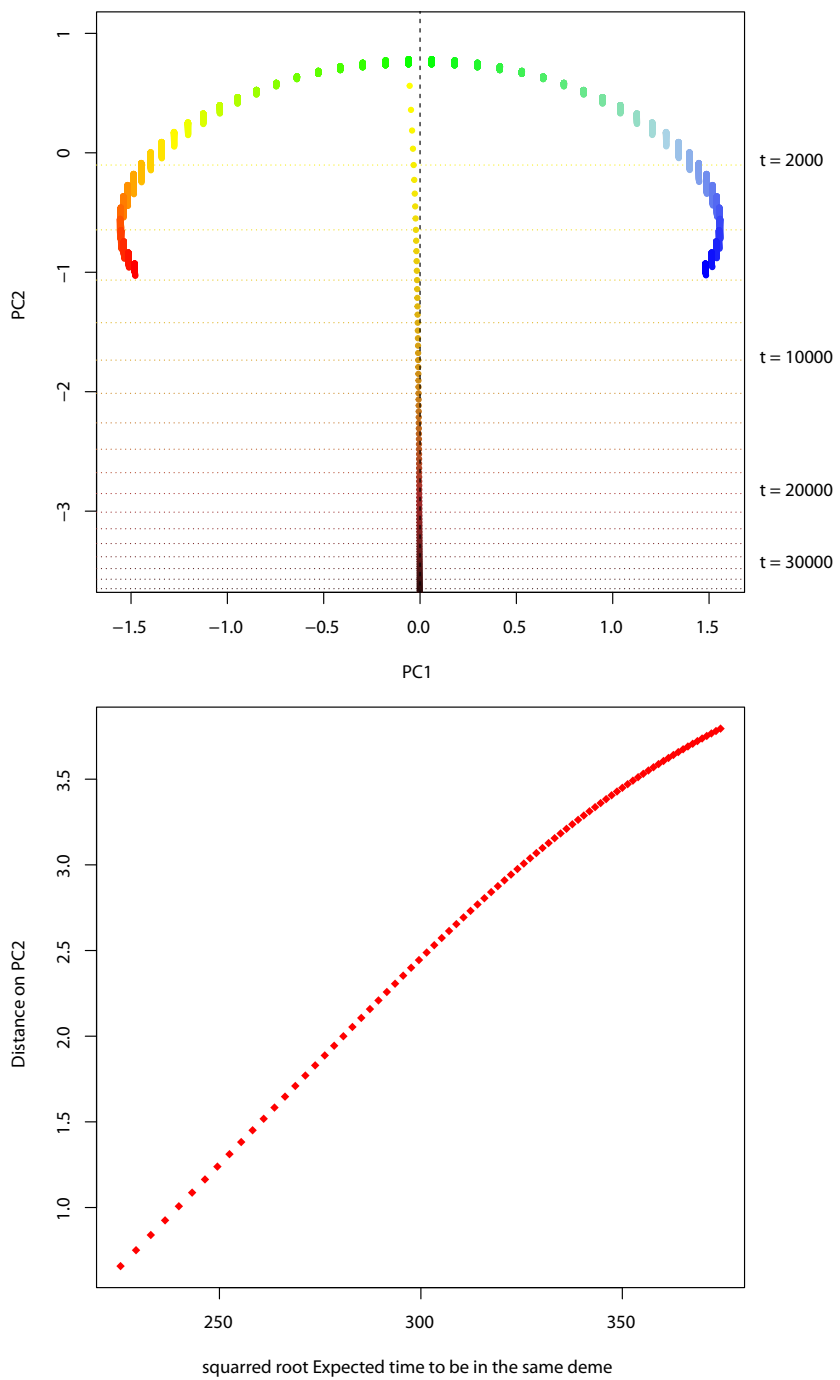

Figure 7: Panel A. Principal components for a 1-Dimensional stepping-stone with 50 present demes, and 1 ancient deme. The PCA is performed several times, with an ancient deme sampled at different times. Panel B. Average distance between present demes and ancient deme on $P C 2$ as a function of $\sqrt{\Delta}$ 
456

\section{Appendix A}

Using the notations in Weiss and Kimura (1965), we calculate the covariance of the allele frequencies $\rho(k)$ between two populations that are spatially separated by $k$ units of distance. This quantity is defined by

$$
\rho(k)=E[\tilde{p}(0) \tilde{p}(k)] .
$$

In the case where the demes are also separated by $t$ units of time, we define

$$
\rho(k, t)=E\left[\tilde{p}(0)^{(0)} \tilde{p}(k)^{(t)}\right] .
$$

and in the particular case of $t=1$,

$$
\begin{aligned}
\rho(k, 1) & =E\left[\tilde{p}(k)^{(1)} \tilde{p}(0)^{(0)}\right] \\
& =E\left[\tilde{p}(k)^{\prime} \tilde{p}(0)\right] \\
& =E[(L \tilde{p}(k)+\epsilon(k)) \tilde{p}(0)] \\
& =E[L \tilde{p}(k) \tilde{p}(0)]+E[L \epsilon(k) \tilde{p}(0)] \\
& =L E[\tilde{p}(k) \tilde{p}(0)] \\
& =L \rho(k) .
\end{aligned}
$$

58 By induction, we show that for any value of $t>0$

$$
\rho(k, t)=L^{t} \rho(k) .
$$

459 Let's assume that for a time $t>0$ equation (24) is true,

$$
\begin{aligned}
\rho(k, t+1) & =E\left[\tilde{p}(k)^{(t+1)} \tilde{p}(0)^{(0)}\right] \\
& =E\left[\left(L \tilde{p}(k)^{(t)}+\epsilon(k)^{(t)}\right) \tilde{p}(0)\right] \\
& =E\left[L \tilde{p}(k)^{(t)} \tilde{p}(0)\right]+E\left[L \epsilon(k)^{(t)} \tilde{p}(0)\right] \\
& =L E\left[\tilde{p}(k)^{(t)} \tilde{p}(0)\right] \\
& =L \rho(k, t) \\
& =L^{t} \rho(k) .
\end{aligned}
$$

461 Then to obtain the correlation of allele frequencies $r(k, t)$ between two 462 demes, we have $\rho(0,0)=\rho(0)$ and

$$
r(k, t)=\frac{\rho(k, t)}{\rho(0,0)}=\frac{L^{t} \rho(k)}{\rho(0)}=L^{t} r(k) .
$$


466

467

471

473

474

$$
\begin{aligned}
& L^{t+1} \cos (k \theta)=L\left[L^{t} \cos (k \theta)\right] \\
& =L\left[\sum_{i_{1}=0}^{\infty} \cdots \sum_{i_{t}=0}^{\infty} m_{i_{1}} \ldots m_{i_{t}} \cos \left(i_{1} \theta\right) \ldots \cos \left(i_{t} \theta\right)\right] \cos (k \theta) \\
& =\sum_{i_{t+1}=0}^{\infty}\left[\sum_{i_{1}=0}^{\infty} \cdots \sum_{i_{t}=0}^{\infty} m_{i_{1}} \ldots m_{i_{t}} \cos \left(i_{1} \theta\right) \ldots \cos \left(i_{t} \theta\right)\right] \\
& \times \frac{m_{i_{t+1}}}{2}\left(\cos \left(\left(k+i_{t+1}\right) \theta\right)+\cos \left(\left(k-i_{t+1}\right) \theta\right)\right) \\
& =\sum_{i_{t+1}=0}^{\infty} \sum_{i_{1}=0}^{\infty} \cdots \sum_{i_{t}=0}^{\infty} m_{i_{1}} \ldots m_{i_{t}} m_{i_{t+1}} \cos \left(i_{1} \theta\right) \ldots \cos \left(i_{t} \theta\right) \cos \left(i_{t+1} \theta\right) \cos (k \theta) \\
& =\left[\sum_{i=0}^{\infty} m_{i} \cos (i \theta)\right]^{t+1} \cos (k \theta) \text {. }
\end{aligned}
$$

475 We can conclude by induction that formula $(26)$ is true for any positive $t$.

476 Then, using equation (26), a general formula for $r(k, t)$ can be expressed

$$
r(k, t)=\frac{C}{2 \pi} \int_{0}^{2 \pi} \frac{\left[\sum_{i=0}^{\infty} m_{i} \cos (i \theta)\right]^{t} \cos (k \theta) d \theta}{1-\left[\sum_{i=0}^{\infty} m_{i} \cos (i \theta)\right]^{2}} .
$$

477 Constant $\mathrm{C}$ is set such that $r(0,0)=1$. We do not analytically inves478 tigate this constant, however details about the case $t=0$ can be found in 479 Weiss and Kimura (1965). 


\section{Appendix C}

Let's assume the particular stepping-stone model: $\sum_{i=0}^{\infty} m_{i} \cos (i \theta)=1-$ $m_{1}-m_{\infty}+m_{1} \cos (\theta)$. Now the correlation between 2 demes $k$ steps appart and $t$ generations is

$$
r(k, t)=\frac{C}{2 \pi} \int_{0}^{2 \pi} \frac{\left[1-m_{1}-m_{\infty}+m_{1} \cos (\theta)\right]^{t} \cos (k \theta) d \theta}{1-\left[1-m_{1}-m_{\infty}+m_{1} \cos (\theta)\right]^{2}} .
$$

The fraction can be decomposed in two parts $r(k, t)=C /(2 \pi)\left(A_{1}(k, t)+\right.$ $\left.A_{2}(k, t)\right)$ using partial fraction expansion, where

$$
\begin{aligned}
& A_{1}(k, t)=\int_{0}^{2 \pi} \frac{\left[1-m_{1}-m_{\infty}+m_{1} \cos (\theta)\right]^{t} \cos (k \theta) d \theta}{1-\left[1-m_{1}-m_{\infty}+m_{1} \cos (\theta)\right]} \\
& A_{2}(k, t)=\int_{0}^{2 \pi} \frac{\left[1-m_{1}-m_{\infty}+m_{1} \cos (\theta)\right]^{t} \cos (k \theta) d \theta}{1+\left[1-m_{1}-m_{\infty}+m_{1} \cos (\theta)\right]}
\end{aligned}
$$

. Let $\alpha=\left(1-m_{1}-m_{\infty}\right) / m_{1}$, we can expand $A_{1}$ and $A_{2}$,

$$
\begin{gathered}
A_{1}(k, t)=-m_{1}^{t-1} \sum_{i=0}^{t}\left(\begin{array}{l}
t \\
i
\end{array}\right) \alpha^{t-i} \int_{0}^{2 \pi} \frac{\cos (\theta)^{i} \cos (k \theta) d \theta}{\alpha-\frac{1}{m_{1}}+\cos (\theta)} \\
A_{2}(k, t)=m_{1}^{t-1} \sum_{i=0}^{t}\left(\begin{array}{l}
t \\
i
\end{array}\right) \alpha^{t-i} \int_{0}^{2 \pi} \frac{\cos (\theta)^{i} \cos (k \theta) d \theta}{\alpha+\frac{1}{m_{1}}+\cos (\theta)}
\end{gathered}
$$

To get rid of the integral, we can use the fact that

$$
\int_{0}^{2 \pi} \frac{\cos ^{t}(\theta) \cos (k \theta) d \theta}{x+\cos (\theta)}=\frac{1}{2^{t}} \sum_{i=0}^{t} a_{i}^{(t)} \int_{0}^{2 \pi} \frac{\cos ((k+i) \theta)+\cos ((k-i) \theta) d \theta}{x+\cos (\theta)}
$$

481

where

482

\begin{tabular}{c|cccccc|c}
$i$ & 0 & 1 & 2 & 3 & 4 & 5 & Sum \\
\hline$a_{i}^{(1)}$ & 0 & 1 & & & & & $2 \times 1=2$ \\
$a_{i}^{(2)}$ & 2 & 0 & 1 & & & & $2 \times 1+2=4$ \\
$a_{i}^{(3)}$ & 0 & 3 & 0 & 1 & & & $2 \times(1+3)=8$ \\
$a_{i}^{(4)}$ & 6 & 0 & 4 & 0 & 1 & & 16 \\
$a_{i}^{(5)}$ & 0 & 10 & 0 & 5 & 0 & 1 & 32
\end{tabular}

483 and as given in Weiss and Kimura (1965) 
484

485

486

487

488

$$
\frac{1}{2 \pi} \int_{0}^{2 \pi} \frac{\cos (k \theta) d \theta}{x+\cos (\theta)}= \begin{cases}\frac{1}{\sqrt{x^{2}-1}}\left(\sqrt{x^{2}-1}-x\right)^{n}, & x>1 \\ \frac{(-1)^{n+1}}{\sqrt{x^{2}-1}}\left(\sqrt{x^{2}-1}+x\right)^{n}, & x<-1\end{cases}
$$

This leads us to the expressions for $A_{1}$ and $A_{2}$,

$$
\begin{array}{r}
A_{1}(k, t)=-m_{1}^{t-1} \sum_{i=0}^{t}\left(\begin{array}{l}
t \\
i
\end{array}\right) \alpha^{t-i} \frac{1}{2^{i}} \sum_{j=1}^{i}\left\{\begin{array}{r}
(i) \frac{(-1)^{k+j}}{\sqrt{\left(\alpha-\frac{1}{m_{1}}\right)^{2}-1}}\left(\alpha-\frac{1}{m_{1}}+\sqrt{\left.\left(\alpha-\frac{1}{m_{1}}\right)^{2}-1\right)^{k+j}}\right. \\
a_{j}^{(i)} \frac{(-1)^{k-j}}{\sqrt{\left(\alpha-\frac{1}{m_{1}}\right)^{2}-1}}\left(\alpha-\frac{1}{m_{1}}+\sqrt{\left.\left.\left(\alpha-\frac{1}{m_{1}}\right)^{2}-1\right)^{k-j}\right\}}\right. \\
A_{2}(k, t)=m_{1}^{t-1} \sum_{i=0}^{t}\left(\begin{array}{l}
t \\
i
\end{array}\right) \alpha^{t-i} \frac{1}{2^{i}} \sum_{j=1}^{i}\left\{\quad a_{j}^{(i)} \frac{1}{\sqrt{\left(\alpha+\frac{1}{m_{1}}\right)^{2}-1}}\left(\sqrt{\left(\alpha+\frac{1}{m_{1}}\right)^{2}-1}-\left(\alpha+\frac{1}{m_{1}}\right)\right)^{k+j}\right. \\
\left.a_{j}^{(i)} \frac{1}{\sqrt{\left(\alpha+\frac{1}{m_{1}}\right)^{2}-1}}\left(\sqrt{\left(\alpha+\frac{1}{m_{1}}\right)^{2}-1}-\left(\alpha+\frac{1}{m_{1}}\right)\right)^{k-j}\right\}
\end{array}\right.
\end{array}
$$




\section{Appendix D}

The 2-Dimensional case of the analysis can be detailed by changing the operators $L$ and $S$. We note the cartesian coordinates of each deme with the couple $\left(i_{1}, i_{2}\right)$, and we define the operators $S_{1}$ and $S_{2}$ such as

$$
S_{1} \tilde{p}\left(i_{1}, i_{2}\right)=\tilde{p}\left(i_{1}+1, i_{2}\right) \text { and } S_{2} \tilde{p}\left(i_{1}, i_{2}\right)=\tilde{p}\left(i_{1}, i_{2}+1\right) .
$$

The operator $L$ in two dimensions becomes

$$
L=\left(1-\sum_{i_{1}} \sum_{i_{2}} m_{i_{1} i_{2}}-m_{\infty}\right) \frac{\left(S_{1}^{0}+S_{2}^{0}\right)}{2}+\sum_{i_{1}} \sum_{i_{2}} \frac{m_{i_{1} i_{2}}}{4}\left(S_{1}^{i_{1}}+S_{1}^{-i_{1}}\right)\left(S_{2}^{i_{2}}+S_{2}^{-i_{2}}\right)
$$

where $m_{i_{1} i_{2}}$ is the migration rate between demes separated by $i_{1}$ and $i_{2}$ steps. The correlation in 2 dimensions can be written using the spectral decomposition and for two demes we have

$$
r\left(k_{1}, k_{2}, 0\right)=\frac{C_{2}}{(2 \pi)^{2}} \int_{0}^{2 \pi} \int_{0}^{2 \pi} \frac{\cos \left(k_{1} \theta_{1}\right) \cos \left(k_{2} \theta_{2}\right) d \theta_{1} d \theta_{2}}{1-\left(\sum_{i_{1}, i_{2}=0}^{\infty} m_{i_{1} i_{2}} \cos \left(i_{1} \theta_{1}\right) \cos \left(i_{2} \theta_{2}\right)\right)^{2}}
$$

for two populations that are separated by $k_{1}$ and $k_{2}$ steps at the same generation. Using the same trigonometric properties as in appendix B, we have

$$
L^{t} \cos \left(k_{1} \theta_{1}\right) \cos \left(k_{2} \theta_{2}\right)=\left[\sum_{i 1} \sum_{i 2}\left(m_{i_{1} i_{2}} \cos \left(i_{1} \theta_{1}\right) \cos \left(i_{2} \theta_{2}\right)\right)\right]^{t} \cos \left(k_{1} \theta_{1}\right) \cos \left(k_{2} \theta_{2}\right)
$$

and $m_{00}=\left(1-\sum_{i_{1}} \sum_{i_{2}} m_{i_{1} i_{2}}-m_{\infty}\right)$. As a consequence, the correlation of allele frequencies in 2 dimensions between two populations separated by $k_{1}$ and $k_{2}$ steps, and $t$ generations is

$r\left(k_{1}, k_{2}, t\right)=\frac{C_{2}}{(2 \pi)^{2}} \int_{0}^{2 \pi} \int_{0}^{2 \pi} \frac{\left[\sum_{i_{1}=0}^{\infty} \sum_{i_{2}=0}^{\infty} m_{i_{1} i_{2}} \cos \left(i_{1} \theta_{1}\right) \cos \left(i_{2} \theta_{2}\right)\right]^{t} \cos \left(k_{1} \theta_{1}\right) \cos \left(k_{2} \theta_{2}\right) d \theta_{1} d \theta_{2}}{1-\left(\sum_{i_{1}=0}^{\infty} \sum_{i_{2}=0}^{\infty} m_{i_{1} i_{2}} \cos \left(i_{1} \theta_{1}\right) \cos \left(i_{2} \theta_{2}\right)\right)^{2}}$.

To go further, and especially investigate the 3-Dimensional case that can be relevant in practice, it is possible to extend the calculations in ndimensional models, where two populations are separated by $t$ generations and a vector of steps $\left(k_{1}, \ldots k_{n}\right)$. Redefining the operators $\mathrm{S}$ and $\mathrm{L}$, we can show that the correlation is

$r\left(k_{1}, \ldots, k_{n}, t\right)=\frac{C_{n}}{(2 \pi)^{n}} \int_{0}^{2 \pi} \ldots \int_{0}^{2 \pi} \frac{\left[\sum_{i_{1}, i_{n}=0}^{\infty} m_{i_{1} \ldots i_{n}} \cos \left(i_{1} \theta_{1}\right) \ldots \cos \left(i_{n} \theta_{n}\right)\right]^{t} \cos \left(k_{1} \theta_{1}\right) \ldots \cos \left(k_{n} \theta_{n}\right) d \theta_{1} .}{1-\left(\sum_{i_{1}, \ldots i_{n}=0}^{\infty} m_{i_{1} \ldots i_{n}} \cos \left(i_{1} \theta_{1}\right) \ldots \cos \left(i_{n} \theta_{n}\right)\right)^{2}}$ 


\section{Appendix E}

491 We detail the case where two groups are present in the data, the present demes and the ancient deme. The quantity $\Delta$ is the time for two genes in different groups to be in the same group. In the case where there is one ancient deme $k_{2}$ and one present deme $k_{1}$, using equation $(19)$ we have

$$
\begin{aligned}
E\left[\Delta \mid k_{1}\right] & =E\left[T_{A_{1} A_{2}}\right]-2 N_{e} n_{d} \\
& =t+\frac{1}{\sqrt{2 \pi m_{1} t}} \int_{0}^{n_{d}}\left(n_{d}-\left|x-k_{2}\right|\right) \frac{\left|x-k_{2}\right|}{2 m_{1}} e^{-\frac{\left(x-k_{1}\right)^{2}}{2 m_{1} t}} d x .
\end{aligned}
$$

In the practical case we consider several present time demes $1 \ldots n_{p}$, and one ancient deme. The expectation of $\Delta$ has to be conditioned by the probability that $A_{1}$ is in a given present population $k_{1}$.

$$
E[\Delta]=\sum_{i=1}^{n_{p}} p\left(k_{1}=j\right) E\left[\Delta \mid k_{1}=j\right] .
$$

Since we consider a stepping-stone model where all the populations have the same effective population size, we have $p\left(k_{1}=j\right)=1 / n_{p}, j=1 \ldots n_{p}$. 


\section{References}

Al-Hassan, Q. (2012). On powers of tridiagonal matrices with nonnegative entries. Journal of Applied Mathematical Sciences, 6(48):2357-2368.

Andrello, M., Bevacqua, D., Maes, G. E., and De Leo, G. A. (2011). An integrated genetic-demographic model to unravel the origin of genetic structure in european eel (anguilla anguilla 1.). Evolutionary applications, 4(4):517-533.

Baird, S. J. and Santos, F. (2010). Monte carlo integration over stepping stone models for spatial genetic inference using approximate bayesian computation. Molecular ecology resources, 10(5):873-885.

Baran, Y. and Halperin, E. (2015). A note on the relations between spatiogenetic models. Journal of Computational Biology.

Barton, N. H., Depaulis, F., and Etheridge, A. M. (2002). Neutral evolution in spatially continuous populations. Theoretical population biology, 61(1):31-48.

Barton, N. H., Etheridge, A. M., and Véber, A. (2010). A new model for evolution in a spatial continuum. Electron. J. Probab, 15(7).

Beaumont, M. A., Zhang, W., and Balding, D. J. (2002). Approximate bayesian computation in population genetics. Genetics, 162(4):2025-2035.

Castric, V. and Bernatchez, L. (2003). The rise and fall of isolation by distance in the anadromous brook charr (salvelinus fontinalis mitchill). Genetics, 163(3):983-996.

Cox, J. T., Durrett, R., et al. (2002). The stepping stone model: New formulas expose old myths. The Annals of Applied Probability, 12(4):13481377 .

Crow, J. F., Kimura, M., et al. (1970). An introduction to population genetics theory. An introduction to population genetics theory.

Csilléry, K., Blum, M. G., Gaggiotti, O. E., and François, O. (2010). Approximate bayesian computation (abc) in practice. Trends in ecology $\&$ evolution, 25(7):410-418.

De, A. and Durrett, R. (2007). Stepping-stone spatial structure causes slow decay of linkage disequilibrium and shifts the site frequency spectrum. Genetics, 176(2):969-981. 
Depaulis, F., Orlando, L., and Hänni, C. (2009). Using classical population genetics tools with heterochroneous data: time matters! PLoS One, $4(5): \mathrm{e} 5541$.

Doob, J. L. (1953). Stochastic processes, volume 101. New York Wiley.

Duforet-Frebourg, N. and Blum, M. G. (2014). Nonstationary patterns of isolation-by-distance: inferring measures of local genetic differentiation with bayesian kriging. Evolution, 68(4):1110-1123.

Duforet-Frebourg, N., Laval, G., Bazin, E., and Blum, M. G. (2015). Detecting genomic signatures of natural selection with principal component analysis: application to the 1000 genomes data. arXiv preprint arXiv:1504.04543.

Durand, E. Y., Patterson, N., Reich, D., and Slatkin, M. (2011). Testing for ancient admixture between closely related populations. Molecular biology and evolution, 28(8):2239-2252.

Engelhardt, B. E. and Stephens, M. (2010). Analysis of population structure: a unifying framework and novel methods based on sparse factor analysis. PLoS Genet, 6(9):e1001117.

Epperson, B. K. (2000). Spatial and space-time correlations in ecological models. Ecological modelling, 132(1):63-76.

Excoffier, L. and Foll, M. (2011). Fastsimcoal: a continuous-time coalescent simulator of genomic diversity under arbitrarily complex evolutionary scenarios. Bioinformatics, 27(9):1332-1334.

Felsenstein, J. (2015). Covariation of gene frequencies in a stepping-stone lattice of populations. Theoretical population biology, 100:88-97.

Haak, W., Lazaridis, I., Patterson, N., Rohland, N., Mallick, S., Llamas, B., Brandt, G., Nordenfelt, S., Harney, E., Stewardson, K., et al. (2015). Massive migration from the steppe was a source for indo-european languages in europe. Nature.

Hallatschek, O., Hersen, P., Ramanathan, S., and Nelson, D. R. (2007). Genetic drift at expanding frontiers promotes gene segregation. Proceedings of the National Academy of Sciences, 104(50):19926-19930.

Hellberg, M. E. (2009). Gene flow and isolation among populations of marine animals. Ecology, Evolution, and Systematic. 
Higuchi, R., Bowman, B., Freiberger, M., Ryder, O. A., and Wilson, A. C. (1984). Dna sequences from the quagga, an extinct member of the horse family. Nature.

Hudson, R. R. (2002). Generating samples under a wright-fisher neutral model of genetic variation. Bioinformatics, 18(2):337-338.

Jay, F., Sjödin, P., Jakobsson, M., and Blum, M. G. (2013). Anisotropic isolation by distance: the main orientations of human genetic differentiation. Molecular biology and evolution, 30(3):513-525.

Karakachoff, M., Duforet-Frebourg, N., Simonet, F., Le Scouarnec, S., Pellen, N., Lecointe, S., Charpentier, E., Gros, F., Cauchi, S., Froguel, P., et al. (2015). Fine-scale human genetic structure in western france. European Journal of Human Genetics, 23(6):831-836.

Kimura, M. (1953). s̈tepping stonem̈odel of population. Ann. Rept. Nat. Inst. Genetics Japan, pages 62-63.

Kimura, M. and Crow, J. F. (1963). The measurement of effective population number. Evolution, pages 279-288.

Kimura, M. and Weiss, G. H. (1964). The stepping stone model of population structure and the decrease of genetic correlation with distance. Genetics, 49(4):561.

Loh, P.-R., Lipson, M., Patterson, N., Moorjani, P., Pickrell, J. K., Reich, D., and Berger, B. (2013). Inferring admixture histories of human populations using linkage disequilibrium. Genetics, 193(4):1233-1254.

Malécot, G. (1948). mathématiques de l'héreédité. Paris: Masson etCie.

Malécot, G. (1955). The decrease of relationship with distance. In Cold Spring Harbor Symp. Quant. Biol, volume 20, pages 52-53.

Maruyama, T. (1970a). Rate of decrease of genetic variability in a subdivided population. Biometrika, 57(2):299-311.

Maruyama, T. (1970b). Stepping stone models of finite length. Advances in Applied Probability, pages 229-258.

Maruyama, T. (1971a). Analysis of population structure: Ii. twodimensional stepping sone models of finite length and other geographically structured populations*. Annals of human genetics, 35(2):179-196. 
Maruyama, T. (1971b). The rate of decrease of heterozygosity in a population occupying a circular or a linear habitat. Genetics, 67(3):437.

Maruyama, T. (1972). Rate of decrease of genetic variability in a twodimensional continuous population of finite size. Genetics, 70(4):639-651.

McVean, G. (2009). A genealogical interpretation of principal components analysis. PLoS Genet, 5(10):e1000686.

Nagylaki, T. (1983). The robustness of neutral models of geographical variation. Theoretical Population Biology, 24(3):268-294.

Nei, M. (1973). Analysis of gene diversity in subdivided populations. Proceedings of the National Academy of Sciences, 70(12):3321-3323.

Novembre, J., Johnson, T., Bryc, K., Kutalik, Z., Boyko, A. R., Auton, A., Indap, A., King, K. S., Bergmann, S., Nelson, M. R., et al. (2008). Genes mirror geography within europe. Nature, 456(7218):98-101.

Novembre, J. and Stephens, M. (2008). Interpreting principal component analyses of spatial population genetic variation. Nature genetics, 40(5):646-649.

Pääbo, S. (1985). Molecular cloning of ancient egyptian mummy dna. $N a-$ ture.

Pääbo, S., Poinar, H., Serre, D., Jaenicke-Després, V., Hebler, J., Rohland, N., Kuch, M., Krause, J., Vigilant, L., and Hofreiter, M. (2004). Genetic analyses from ancient dna. Annu. Rev. Genet., 38:645-679.

Patterson, N., Price, A. L., and Reich, D. (2006). Population structure and eigenanalysis. PLoS Genetics.

Peter, B. M. and Slatkin, M. (2013). Detecting range expansions from genetic data. Evolution, 67(11):3274-3289.

Petkova, D., Novembre, J., and Stephens, M. (2014). Visualizing spatial population structure with estimated effective migration surfaces. bioRxiv, page 011809.

Pickrell, J. K. and Reich, D. (2014). Toward a new history and geography of human genes informed by ancient dna. Trends in Genetics, 30(9):377-389. 
Ramachandran, S., Deshpande, O., Roseman, C. C., Rosenberg, N. A., Feldman, M. W., and Cavalli-Sforza, L. L. (2005). Support from the relationship of genetic and geographic distance in human populations for a serial founder effect originating in africa. Proceedings of the National Academy of Sciences of the United States of America, 102(44):15942-15947.

Ross, S. M. et al. (1996). Stochastic processes, volume 2. John Wiley \& Sons New York.

Rousset, F. (1997). Genetic differentiation and estimation of gene flow from f-statistics under isolation by distance. Genetics, 145(4):1219-1228.

Sharbel, T. F., Haubold, B., and Mitchell-Olds, T. (2000). Genetic isolation by distance in arabidopsis thaliana: biogeography and postglacial colonization of europe. Molecular Ecology, 9(12):2109-2118.

Skoglund, P., Malmström, H., Raghavan, M., Storå, J., Hall, P., Willerslev, E., Gilbert, M. T. P., Götherström, A., and Jakobsson, M. (2012). Origins and genetic legacy of neolithic farmers and hunter-gatherers in europe. Science, 336(6080):466-469.

Skoglund, P., Sjödin, P., Skoglund, T., Lascoux, M., and Jakobsson, M. (2014). Investigating population history using temporal genetic differentiation. Molecular biology and evolution, 31(9):2516-2527.

Slatkin, M. (1985). Gene flow in natural populations. Annual review of ecology and systematics, pages 393-430.

Slatkin, M. (1991). Inbreeding coefficients and coalescence times. Genetical research, 58(02):167-175.

Slatkin, M. (1993). Isolation by distance in equilibrium and non-equilibrium populations. Evolution, pages 264-279.

Teacher, A. G., Thomas, J. A., and Barnes, I. (2011). Modern and ancient red fox (vulpes vulpes) in europe show an unusual lack of geographical and temporal structuring, and differing responses within the carnivores to historical climatic change. BMC evolutionary biology, 11(1):214.

Weir, B. S. and Cockerham, C. C. (1984). Estimating f-statistics for the analysis of population structure. evolution, pages 1358-1370.

Weiss, G. H. and Kimura, M. (1965). A mathematical analysis of the stepping stone model of genetic correlation. Journal of Applied Probability, pages 129-149. 
${ }_{663}$ Wilkins, J. F. and Wakeley, J. (2002). The coalescent in a continuous, finite,

${ }_{664}$ linear population. Genetics, 161(2):873-888.

665 Wright, S. (1940). Breeding structure of populations in relation to specia-

666 tion. American Naturalist, pages 232-248.

667 Wright, S. (1943). Isolation by distance. Genetics, 28(2):114. 